\title{
Trivium
}

Revue franco-allemande de sciences humaines et sociales - Deutsch-französische Zeitschrift für Geistesund Sozialwissenschaften

$33 \mid 2021$

Concepts historiques fondamentaux - Démocratie

\section{Démocratie. Présentation}

\section{Catherine Colliot-Thélène}

\section{OpenEdition}

Journals

Édition électronique

URL : https://journals.openedition.org/trivium/7734

DOI : $10.4000 /$ trivium.7734

ISSN : 1963-1820

Éditeur

Les éditions de la Maison des sciences de l'Homme

\section{Référence électronique}

Catherine Colliot-Thélène, « Démocratie. Présentation », Trivium [En ligne], 33 | 2021, mis en ligne le 07 juin 2021, consulté le 10 juin 2021. URL : http://journals.openedition.org/trivium/7734 ; DOI : https:// doi.org/10.4000/trivium.7734

Ce document a été généré automatiquement le 10 juin 2021.

\section{c) (7) $\Theta$}

Les contenus des la revue Trivium sont mis à disposition selon les termes de la Licence Creative Commons Attribution - Pas d'Utilisation Commerciale - Pas de Modification 4.0 International. 


\title{
Démocratie. Présentation
}

\author{
Catherine Colliot-Thélène
}

1 Le texte ici présenté est la traduction d'un premier article tiré des Geschichtliche Grundbegriffe $^{1}(G G)$, qui sera suivie par la traduction des articles "Herrschaft » (La domination), « Macht-Gewalt » (Pouvoir/violence) et « Staat und Souveränität » (État et souveraineté). Au total, ce seront quatre articles d'une encyclopédie qui en comprend en tout 122. La longueur des entrées de cette encyclopédie sans pareille, généralement comparable à celle d'un gros article, voire dans certains cas d'un petit livre, explique qu'il soit impossible d'envisager une traduction de la totalité de l'ouvrage. Il fallait donc faire un choix.

Celui que nous proposons ici n'est pas aléatoire: nous avons sélectionné des articles thématiquement fortement liés, et qui concernent des concepts qui sont aujourd'hui encore (ou peut-être à nouveau ?) au cœur des débats politiques et des théories de la politique, qu'elles relèvent de la philosophie, des sciences politiques ou d'autres disciplines. Si la parenté thématique entre les articles "Domination", "Pouvoir/ violence » et "État/souveraineté » est suffisamment évidente pour se passer de commentaire - tous traitent de la dimension verticale de la politique, de ce par quoi elle évoque des relations hiérarchiques, ou plus généralement inégalitaires entre des individus et des groupes d'individus dont certains commandent et d'autres obéissent -, l'adjonction du concept de "démocratie " à cette sélection introduit une dissonance. Malgré les disputes largement ouvertes aujourd'hui sur ce que le terme démocratie désigne précisément, il y a en effet un semblant de consensus sur l'antinomie entre démocratie d'un côté, domination et violence de l'autre. Si l'on fait exception des anarchistes, la plupart des gens considèrent que la démocratie est compatible avec l'État (les États occidentaux se flattent d'être des «États démocratiques ») ainsi qu'avec la souveraineté, dès lors que celle-ci est spécifiée comme populaire, certains allant même jusqu'à identifier démocratie et souveraineté populaire. Beaucoup aussi veulent bien reconnaître que la démocratie est une forme de pouvoir, mais c'est à la condition de distinguer nettement entre pouvoir et domination ${ }^{2}$. La notion de démocratie, depuis l'Antiquité jusqu'à nos jours, entretient un rapport étroit avec l'égalité, et elle entre pour cette raison en contradiction avec une conception de la politique qui insiste sur la dissymétrie irréductible dans toute relation de pouvoir, et en particulier dans les 
relations constitutives de ce que nous nommons la politique. Mais c'est précisément en raison de cette tension qu'il est apparu pertinent d'inclure dans notre choix d'articles celui relatif à la démocratie. Si la démocratie, loin d'être une évidence, a longtemps été au contraire un problème pour la philosophie et la théorie politique ${ }^{3}$, un des paradoxes de l'histoire de la pensée politique occidentale est qu'elle soit devenue de nos jours le nom du bon régime en général, ou du moins une valeur quasiment incontestée. À suivre l'histoire des concepts de domination, de pouvoir et de violence, d'État et de souveraineté, et, en contrepoint, celle du concept de démocratie, on entrevoit les raisons de ce paradoxe: la valorisation croissante de la démocratie, à partir de la seconde moitié du XIX ${ }^{\mathrm{e}}$ siècle, et plus encore après la Seconde Guerre mondiale, est allée de pair avec la condamnation des différentiels de pouvoir au sein des sociétés qui se disent démocratiques, des différentiels dont on ne peut certes nier l'existence, mais que l'on neutralise en les justifiant par des nécessités fonctionnelles. Au point que l'on doit se demander si la quasi-unanimité qui règne aujourd'hui, dans le monde occidental ${ }^{4}$, concernant les mérites de la démocratie n'a pas pour effet, voire pour fonction principale d'occulter la permanence de phénomènes qui relèvent bien de la domination, voire de la violence, dans toutes les figures de la politique : des constantes, certes diversement modalisées (plus ou moins intenses ou atténuées, sauvages ou domestiquées), qui transparaissent à travers l'historicisation des concepts.

3 Les considérations qui précèdent ont une portée générale: on constate bien des similitudes entre les histoires des concepts de démocratie, pouvoir, domination, violence, État, souveraineté (ou de leurs équivalents ordinaires dans des langues autres que le français) dans les différents univers langagiers qui cohabitent en Europe (en y incluant leur rejeton étatsunien). Mais une histoire de la pensée politique qui s'accroche aux termes mêmes et aux mutations de leur sens, une histoire intimement liée à une "sémantique historique", a nécessairement un caractère national. Les représentations et théorisations appartenant à d'autres univers langagiers sont pris en compte parce que ces univers langagiers n'étaient pas isolés les uns des autres et que les histoires sociales et politiques des pays concernés ont été depuis l'Antiquité étroitement liées, jusqu'aux bouleversements induits par les révolutions économiques et politiques des Temps modernes, qui les ont tous affectés. Une histoire de la pensée politique en Allemagne en passe nécessairement par Jean-Jacques Rousseau, et en particulier par son Contrat Social, de même qu'une histoire de la pensée politique en France ne peut éviter de faire une place, habituellement large, à Kant. Ou encore, autre exemple, ce n'est pas seulement en France, mais aussi en Allemagne, que De la Démocratie en Amérique de Tocqueville a contribué à conférer un sens social, et non plus seulement politique, à la notion de démocratie. Ces histoires entremêlées et ces expériences partagées ne réduisent pas les différences, souvent importantes ${ }^{5}$, entre pays et entre langues. Le lecteur français en prendra la mesure sur les notions que nous présentons. Dans la courte préface qui introduit le volume VII des $G G^{6}$ (le dernier volume des notions, les deux volumes suivants étant consacrés aux index), Koselleck jetait un regard rétrospectif sur l'entreprise qui se terminait avec la publication de ce volume, en 1992. Il y faisait brièvement allusion à certaines objections qui avaient été formulées à propos de la conception des $G G$ et reconnaissait des insuffisances, au demeurant inévitables (s'agissant notamment de la complétude ${ }^{7}$ ). Il évoquait aussi l'intérêt qu'il y aurait à se pencher sur les procès de traduction "pour découvrir de façon plus exacte et mesurer historiquement les éléments communs et les lignes de séparation entre les langues nationales européennes ${ }^{8} »$. 
4 Nous aurons l'occasion de souligner les "lignes de séparation", et les difficultés de traduction qui leur sont liées, à propos des concepts de Herrschaft [domination], de Macht [pouvoir] et de Gewalt [violence]'. S'agissant de Demokratie, la situation paraît plus simple, le mot s'étant transmis du grec au latin jusqu'aux langues européennes modernes, de sorte qu'il est le même, à la différence de la graphie près, en français et en allemand. On peut s'attendre à de plus grandes similitudes entre les histoires allemande et française du concept. Une histoire française du concept de démocratie inspiré du modèle des $G G$ serait sans doute assez proche de celle, allemande, que nous présentons ici, en ce qui concerne l'Antiquité et, probablement aussi la période médiévale. La théorie française de la démocratie va elle aussi chercher ses sources chez les historiens et philosophes de la Grèce antique, et le terme ne survit, tout au long du Moyen Âge et jusqu'à la veille de la Révolution, pour l'essentiel, que dans la littérature érudite, comme l'une des trois formes de gouvernement de la typologie aristotélicienne. Malgré Rousseau ${ }^{10}$, volontiers présenté aujourd'hui comme l'un des plus éminents théoriciens de la démocratie, le terme « démocratie » était encore d'un emploi rare durant la Révolution française ${ }^{11}$. Mais des différences entre les trajectoires allemande et française apparaissent à partir du moment où l'usage du terme "démocratie» et de ses corrélats ("démocrate", "démocratique ", "démocratisation») se répand dans la langue commune, soit pour désigner des régimes politiques existants, soit à titre d'idéal qui devrait orienter les réformes et révolutions politiques, et qu'il devient ainsi un point de cristallisation des divergences politiques et des conflits sociaux. Certes, en gros, le basculement de l'usage purement savant du terme «démocratie» à un emploi large, s'appliquant à l'époque contemporaine, a lieu à la même époque, dans les premières décennies du XIX siècle, et plus nettement encore, en Allemagne, autour de 1848. Dans les usages allemands et français du terme démocratie, ainsi que dans le monde anglophone, on cesse d'entendre par démocratie avant tout la démocratie "pure» (directe), limitée par conséquent à des petites entités politiques, et l'opposition entre démocratie, aristocratie et monarchie perd en importance, ainsi que celle, plus récente, entre démocratie et république, à mesure que s'impose l'idée d'une démocratie représentative. Et, en France comme en Allemagne, on voit aussi apparaître dans la seconde moitié du XIX ${ }^{\mathrm{e}}$ siècle, parallèlement au développement de la question sociale, une conception radicale de la démocratie, opposée à la démocratie libérale «bourgeoise ». Elle est à l'origine de la formation d'un parti social-démocrate dont l'importance ne cessa de s'accroître dans le paysage politique de l'Allemagne au début $\mathrm{du} \mathrm{XX}^{\mathrm{e}}$ siècle. L'hostilité à la démocratie, entendue comme régime politique, est restée néanmoins plus profonde et plus largement répandue en Allemagne qu'en France, du fait notamment de la persistance des différences statutaires ainsi que de la monarchie jusqu'en 1918. Les tentatives récurrentes d'accommodement entre démocratie et monarchie étonneront sans doute le lecteur français, de même que la généalogie de la démocratie imaginée par certains auteurs allemands du XIX siècle, qui incluait une composante proprement germanique, non pas seulement l'influence de la Réforme, mais aussi « l'ancienne liberté germanique », remontant à l'époque pré-féodale. Mais la spécificité germanique a pu être aussi revendiquée dans une intention très différente : durant la Première Guerre mondiale, et plus encore ensuite dans les partis d'extrême droite, le nationalisme allemand s'est souvent défini contre la démocratie « occidentale». 
On ne peut bien sûr épuiser, dans ces quelques pages d'introduction, tous les points de comparaison intéressants que la lecture de cette histoire allemande du concept de démocratie suggérera aux lecteurs français. Signalons cependant un aspect qui touche à une thèse centrale des Geschichtliche Grundbegriffe, concernant le lien entre les transformations que connaissent les concepts fondamentaux de la pensée politique durant "l'époque charnière » qui va du milieu du XVIII à la seconde partie du $\mathrm{XIX}^{\mathrm{e}}$ siècle $^{12}$ et la conception nouvelle de l'historicité qui constitue le cœur de ces transformations. En France comme en Allemagne, la valorisation de la démocratie, comme réalité présente ou comme idéal régulateur, a certes partie liée avec la représentation d'un progrès global, scientifique, technique, industriel, mais aussi éthique (la réduction des inégalités, statutaires ou autres, en étant l'une des composantes). Mais la philosophie de l'histoire n'a pas exercé en France une influence aussi importante que ce fut le cas en Allemagne, à en croire l'article que nous traduisons ici. Les spéculations concernant la question de savoir si la démocratie était une forme passée, c'est-à-dire primitive, d'organisation politique, ou bien si elle devait être considérée comme une promesse d'avenir, aussi bien que son interprétation comme « concept de mouvement », n'ont occupé qu'une place marginale par rapport à l'identification simple entre démocratie et souveraineté populaire, laquelle est parue assurée avec l'instauration du suffrage universel ${ }^{13}$. C'est peut-être de nos jours, quand l'évidence du progrès est battue en brèche (par le désastre écologique, la montée en puissance des gouvernements autoritaires dans le monde, la fragilité des gouvernements démocratiques face aux risques perçus comme nouveaux, etc.), que le sens de la démocratie tend à s'identifier à un mouvement, la démocratisation, un mouvement jamais achevé et dont la direction n'a rien de garanti : on évoque aussi bien aujourd'hui, souvent, un processus de « dé-démocratisation ».

\section{BIBLIOGRAPHIE}

Arendt, H. (1972) : Du mensonge à la violence, Paris : Calmann-Lévy.

Bernardi, B. (1999) : La démocratie, Paris : Flammarion.

Brunner, O. / Conze, W. / Koselleck, R. (2004 [1972-1997]): Geschichtliche Grundbegriffe. Historisches Lexikon zur politisch-sozialen Sprache in Deutschland, Stuttgart : Klett-Cotta.

Cassin, B. (2004) : Vocabulaire européen des philosophies. Dictionnaire des intraduisibles, Paris : Le Seuil / Le Robert.

Habermas, J. (1998) : L'intégration républicaine. Essais de théorie politique, trad. R. Rochlitz, Paris :

Fayard.

Manin, P. (1995) : Principes du gouvernement représentatif, Paris : Flammarion.

Rosanvallon, P. (2000) : La démocratie inachevée. Histoire de la souveraineté du peuple en France, Paris : Gallimard. 


\section{NOTES}

1. Cf. l'introduction générale.

2. Ainsi Hannah Arendt, s'opposant sur ce point à Max Weber (Arendt [1972], p. 135 sq.); dans le même sens, Jürgen Habermas soutient qu'avec la transformation de la domination en autolégislation, chez Rousseau et Kant, «la domination politique perd son caractère de force naturelle ; il s'agit d'extirper de la puissance de l'État tout résidu de violentia » (Habermas [1998], p. 72).

3. Dans l'introduction au recueil de textes qu'il a publiés et commentés sous le titre La démocratie (Bernardi [1999]), Bruno Bernardi note (p.15) cette «réalité troublante »: « une anthologie de textes philosophiques sur la démocratie est avant tout une anthologie des critiques possibles de la démocratie ».

4. S'il a été question récemment, à propos des réactions politiques à la pandémie du Coronavirus, de la supériorité possible des régimes autoritaires sur les régimes démocratiques, c'était du point de vue de l'efficacité, et non d'un point de vue normatif. Il est vrai que, pour certains, l'efficacité (dont les critères restent à déterminer) tient lieu de principe normatif.

5. Au point que l'on peut parler dans certains cas d'« intraduisibles », pour reprendre le soustitre $\mathrm{du}$ dictionnaire philosophique réalisé sous la direction de Barbara Cassin (Vocabulaire européen des philosophies. Dictionnaire des intraduisibles, Paris: Le Seuil / Le Robert, 2004). [Cf. à ce sujet l'article de Barbara Cassin dans Trivium, 15, 2013 : "Penser en langues. Présentation du Vocabulaire européen des philosophies "; NDLR].

6. Brunner / Conze / Koselleck (2004), vol. 7, p. V-VIII.

7. Il listait toute une série de notions qui mériteraient d'être analysées dans la perspective de l'histoire conceptuelle: des concepts fondamentaux éthiques et sociaux, tels que «Dienst » (le service), «Glück» (le bonheur), «Pflicht» (le devoir), "Ordnung » (l'ordre), «Treue » (la fidélité), "Tugend» (la vertu), ou encore des concepts relatifs aux institutions ou au groupes sociaux, « Kirche » und "Sekte » (église et secte), "Kunst » (l'art), "Wissenschaft und Technik » (la science et la technique), etc. La liste est évidemment infinie, la complétude inatteignable.

8. Brunner / Conze / Koselleck (2004), vol. 7, p. VIII.

9. Les traductions, données ici entre crochets pour la commodité du lecteur francophone, ne vont précisément pas de soi, comme il sera expliqué dans les présentations de ces notions.

10. Il faudrait ajouter aussi : «malgré Spinoza », mais la philosophie de Spinoza n'a pas influencé les acteurs de la politique d'une manière comparable au Contrat Social de Jean-Jacques Rousseau.

11. Cf. Rosanvallon (2000), p. 23 : «Si tout s'organise autour de souveraineté du peuple, on ne parle d'abord guère de démocratie pour caractériser le régime politique. Le terme n'appartient pas à la langue de 1789.»

12. Cf. l'introduction générale.

13. Cf. Manin (1995), p. 171. Si le gouvernement représentatif a tout d'abord été pensé en France, chez la plupart de ses défenseurs, comme un antidote à la démocratie, l'extension du droit de suffrage, la disparition des cens d'éligibilité et tout particulièrement « l'avènement spectaculaire du suffrage universel » ont donné « une puissante impulsion à la croyance que le gouvernement représentatif se muait peu à peu en démocratie». 
INDEX

Schlüsselwörter : Demokratie

Mots-clés : démocratie

\section{AUTEUR}

\section{CATHERINE COLLIOT-THÉLÈNE}

Catherine Colliot-Thélène est professeur émérite de philosophie à l'Université de Rennes I. Pour plus d'informations, voir la notice suivante. 